\title{
Clinical Aspects: Focusing on Key Unique Organ-Specific Issues of Renal Transplantation
}

\author{
Sindhu Chandran ${ }^{1}$ and Flavio Vincenti ${ }^{2}$ \\ ${ }^{1}$ Division of Nephrology, Department of Medicine, Connie Frank Transplant Center at UCSF, San Francisco, \\ California 04143 \\ ${ }^{2}$ Department of Medicine and Department of Surgery, Deborah Faiman Endowed Chair in Kidney \\ Transplantation, UCSF, San Francisco, California 04143 \\ Correspondence: flavio.vincenti@ucsfmedctr.org
}

Kidney transplantation is the treatment of choice for patients with end-stage renal disease because of improved patient survival and quality of life as compared with dialysis. Successful transplantation requires the prompt recognition and appropriate management of both the immediate posttransplant surgical and medical complications as well as subsequent issues like recurrent disease and chronic rejection that affect long-term graft survival. Guidelines for understanding and managing some of the more important early and late kidney-specific transplant problems, including urologic complications, delayed graft function, acute and chronic rejection, BK polyoma virus infection, and recurrent glomerular disease, are reviewed.

Since the first successful kidney transplant in 1954, kidney transplantation has evolved to become the preferred therapeutic modality for patients with end-stage renal disease, conferring superior patient survival and quality of life as compared with dialysis. Over the past 15 years, 90-d, 6-mo, and 1-, 3-, and 5-yr results have shown ongoing improvement for both living and deceased donor kidney transplant recipients, and the 2011 Annual Data Report of the Scientific Registry of Transplant Recipients suggested for the first time an improvement in 10yr results (Annual Data Report 2011). These improvements have been driven by a decrease in the rate of graft failure and return to dialysis. Three-month and 1-yr graft survival for patients receiving a kidney transplant from a de- ceased donor in 2010 were $96.2 \%$ and $92.9 \%$, respectively.

As of June 30, 2011, 164,200 adults in the United States were surviving with a functioning kidney graft, about twice as many as a decade earlier. These patients present the physician with a complex set of medical issues that require intensive management to allow for longevity of both the patient and the allograft. The evaluation and management of the more commonly encountered post-kidney transplant problems are best organized by the time posttransplantation. Most acute events occur within the first $3 \mathrm{mo}$, and patients are usually clinically stable after the end of the first year. Early issues occurring within the first year are usually recognized and managed by the transplant physicians. Suc-

Editors: Laurence A. Turka and Kathryn J. Wood

Additional Perspectives on Transplantation available at www.perspectivesinmedicine.org

Copyright (C) 2014 Cold Spring Harbor Laboratory Press; all rights reserved; doi: 10.1101/cshperspect.a015644

Cite this article as Cold Spring Harb Perspect Med 2014;4:a015644 


\section{S. Chandran and F. Vincenti}

cessful management of subsequent issues requires heightened vigilance on the part of the community nephrologists who are often taking care of these patients, combined with prompt responses and appropriate interventions by the transplant center.

\section{THE FIRST 3 MONTHS}

Most kidney transplant recipients are discharged home before the end of the first week. The bladder catheter is usually removed before discharge and patients are voiding spontaneously. Graft function is closely monitored and usually still improving. Immunosuppressive therapy is adjusted frequently based on drug levels and the presence of side effects.

\section{Urologic Issues \\ Urinary Retention}

Urinary retention occurs commonly owing to prostatic enlargement or neurogenic bladder, because these diagnoses can be missed if recipients were anuric before the surgery. If the postvoid residual urine volume is $>100 \mathrm{~mL}$, the catheter may need to be replaced and the patient may need training in self-catheterization. $\alpha$ Blocking agents such as tamsulosin or terazosin are often started in older male recipients before catheter removal. Ideally, patients with suspected urologic issues should undergo a pretransplant urologic evaluation.

\section{Urine Leak}

Urinary complications are the most frequent technical adverse events following renal transplantation. In comparative analyses, the incidence of urine leak is $0 \%-9.3 \%$ (Pleass et al. 1995; Veale et al. 2007). Most urine leaks are ureteral rather than vesical in origin because most surgeons now use an extravesical ureteroneocystostomy for ureteral implantation, resulting in a shorter ureter, decreased likelihood of ischemia, and a limited cystotomy that rarely leads to leakage from the bladder. Early urine leaks can be of two types. Those occurring with- in the first 1-4 d are usually related to technical problems with the implantation such as excessive tension at the anastomosis. The second type, presenting 5-10 d posttransplantation, is due to distal ureteral ischemia. The sole arterial supply of the transplant ureter is the descending branch of the main renal artery or its lower polar branch. Damage to these vessels can be sustained during organ recovery, with any trivial mishandling of the ureter jeopardizing the tiny periureteral arterial branches and threatening the anastomosis. It is not always possible to discern intraoperatively whether a ureter will develop ischemic necrosis or stricture.

Clinically, urine leaks often present with excruciating abdominal pain, decreased urine output, and a rise in serum creatinine. Ultrasound may show a fluid collection and hydronephrosis or hydroureter, but not always. Aspiration of the fluid and measurement of fluid creatinine is one method of diagnosis. A Mag3 renogram (Fig. 1) is often helpful but can be falsely negative in the case of sluggish/delayed graft function or if the bladder is decompressed by a Foley catheter. Urine leaks can be treated conservatively, in which case a perinephric drain, ureteral stent, and bladder catheter are left in place until the

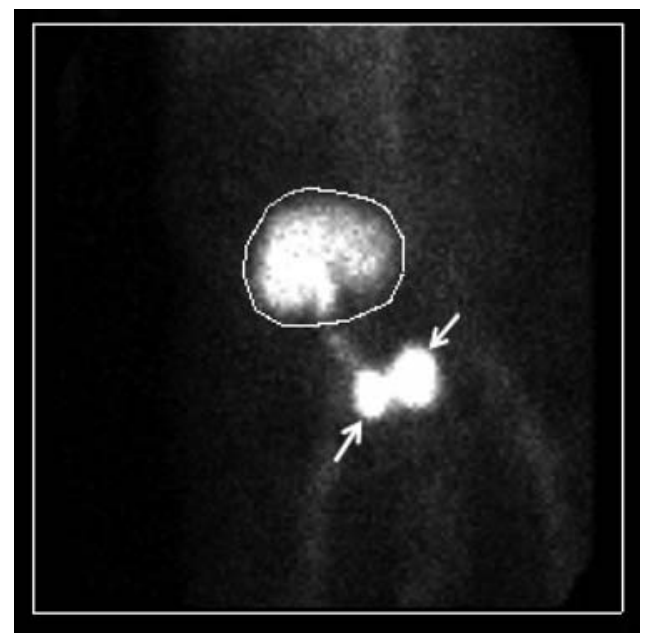

Figure 1. Diagnosis of a urine leak. Mag3 renogram shows a urine leak (top arrow) near the ureterovesical anastomosis. The isotope tracer has been excreted by the kidney (circle) and the bladder is contracted (bottom arrow). 
Renal Transplantation

leak appears to have healed. If the leak persists or is considered unlikely to heal, then reexploration is indicated to reimplant the ureter into the bladder or perform a uretero-ureterostomy to one of the native ureters.

\section{Ureteral Obstruction}

Ureteral obstruction can present early or subsequently after kidney transplantation, with reported incidence rates of $1 \%-8.3 \%$ (Veale et al. 2007). Although some cases of ureteral obstruction are caused by edema, fluid collections, adhesions, tumor, stones, blood clots, or compression by the round ligament or the spermatic cord, are due mostly to strictures, which are believed to be related to ischemia or to rejection events. Potential technical causes of obstruction are kinking within the submucosal tunnel or delayed necrosis of ureteral tips from an excessively tight closure at the seromuscular layer of the bladder. The diagnosis is usually suggested by an ultrasound showing hydronephrosis and/or hydroureter (Fig. 2A) and confirmed by an antegrade pyelogram (Fig. 2B). BK viral infections have also been implicated in the development of subsequent ureteral strictures. Early cases occurring within the first few weeks after transplantation are often managed with a ureteral stent and may not recur after the stent is removed. Recurrent or subsequent cases usually need ureteral revision.

\section{Delayed Graft function}

There are more than 10 definitions of delayed renal allograft function or DGF recorded in the literature based on a range of clinical criteria. Most commonly, DGF has been defined as the use of dialysis within $7 \mathrm{~d}$ of the transplant. This definition has shortfalls because dialysis may be used in the first week after transplant without confirmation of kidney damage. Nevertheless, it offers a standard by which center-specific outcomes can be reported, and epidemiologic analyses and intercenter comparisons performed.

The reported incidence of DGF in deceased donors increased from $14.7 \%$ between 1985 and 1992 to $21.3 \%$ in 2008 despite the progress in acute rejection treatment, and occurred contemporaneously with the use of expanded criteria donors (ECDs) and donation after cardiac death (DCD) (Siedlecki et al. 2011). Most cases are due to ischemic acute tubular necrosis (ATN). Other causes include accelerated cellular or humoral rejection, vascular compromise, urinary obstruction or urine leak, nephrotoxicity from calcineurin inhibitors $(\mathrm{CNI})$, and thrombotic microangiopathy. Donor-specific risk factors for DGF include both immunologic (e.g., absence of T-cell antibody induction therapy, female donor) and nonimmunologic (donor age, weight, prolonged cold/warm ischemia time) factors. Distinct recipient characteristics also confer risk of DGF. Maintenance dialysis
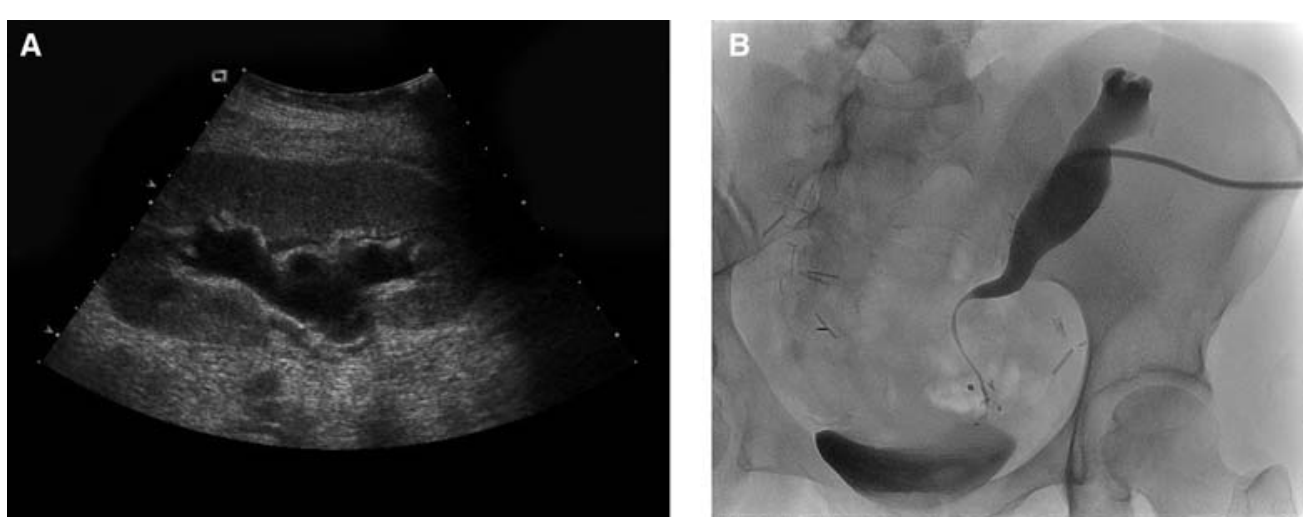

Figure 2. Diagnosis of ureteral obstruction. (A) Transplant renal ultrasound shows hydronephrosis. (B) Antegrade pyelogram in the same patient shows distal long segment ureteral stricture. 
before transplant is perhaps the single greatest contributor. Other factors include obesity, diabetes, age $>55 \mathrm{yr}$, male sex, African American race, prolonged wait period, presensitization, and small-for-size organs. Many of these donor and recipient characteristics are included in a nomogram used to obtain a composite risk assessment before the initiation of implantation surgery (Irish et al. 2010).

After competing prerenal, renal, and postrenal diagnoses are excluded, patients suspected of DGF should preferably be biopsied 5-14 d posttransplantation to rule out acute rejection as a cause of graft dysfunction. DGF associated with ischemic ATN is characterized by ischemia-reperfusion injury and immunologic dysregulation. Endothelial injury has been shown to up-regulate the expression of donor HLA antigens, adhesion molecules, and costimulatory molecules, thus increasing the risk for acute rejection.

Recent outcome studies have not shown a negative effect of DGF on long-term graft survival in the absence of acute rejection (Kayler et al. 2011). However, DGF consumes financial and programmatic resources in terms of prolonged hospitalization, dialysis treatments, and the higher utilization of radiologic studies and biopsies. The effect of supportive parameters on reducing the duration of DGF remains unknown. Posttransplant hemodialysis should be offered when clinically indicated. Hemodynamic instability and nephrotoxins should be avoided. It is important to achieve and maintain adequate immunosuppression in the presence of ATN. Although it has been theorized that a delay in CNI introduction might shorten the course of DGF by avoiding nephrotoxicity, this is unproven and the long-term benefits of such an approach remain debatable. Use of antilymphocyte therapy after DGF is established may not treat the DGF, but will reduce the rejection rate and minimize the negative impact of acute rejection in association with DGF.

The complexity of pathological mechanisms that cause DGF offers many potential targets for therapy to inhibit oxidant stress, encourage vasodilation, and blunt the immune response. There are several ongoing clinical tri- als currently exploring different targets with the primary end point of DGF.

\section{Acute Rejection}

With the use of potent induction and maintenance immunosuppressive agents, the incidence of acute rejection within the first year following transplantation has fallen dramatically, with current rates $<15 \%$ in most centers. When acute rejection occurs, it remains an important clinical problem, increasing the relative risk for early transplant failure as well as contributing to the development of chronic rejection and late graft loss.

Acute rejection in kidney transplants is suspected whenever there is a rise in serum creatinine and is diagnosed by a kidney biopsy. The Banff Classification of Renal Allograft Pathology was originally developed in 1991 and was merged together with the most useful parts of the CCTT classification in 1997, to provide the framework on which today's schema is built. Criteria for antibody-mediated rejection were added in 2003 and updated in 2007 (Solez et al. 2008).

Current markers for acute rejection are imperfect. A rise in serum creatinine lacks sensitivity and specificity for the diagnosis of acute rejection. A kidney biopsy, on the other hand, is invasive and its interpretation subject to reader bias as well as sampling error. Newer assays that look at biomarkers in blood and urine are less invasive and may yield earlier and more precise measurements. Gene expression patterns in plasma, peripheral blood lymphocytes, and biopsy tissue using techniques such as PCR and DNA microarray are currently being evaluated to detect and distinguish different types of rejection. Lack of regulatory T cells (or Tregs) during acute rejection results in unimpeded effector T-cell activity and impaired graft function. Urine levels of the mRNA for the FOXP3 gene, a transcription factor for Tregs, have been found to be positively correlated with graft survival (Muthukumar et al. 2005). A urine test for FOXP3 has been commercially developed and was released in 2012 for limited clinical use. In another landmark study ( $\mathrm{Li}$ et al. 2012), a five- 
gene signature in peripheral blood lymphocytes composed of DUSP1, PBEF1, PSEN1, MAPK9, and NKTR was found to diagnose acute rejection with $91 \%$ sensitivity and $94 \%$ specificity in a largely pediatric population. Although this signature needs validation in larger and more diverse cohorts as well as correlation with graft outcome, it holds great potential as a diagnostic tool that can add to or replace the kidney biopsy as well as monitor the response to therapy.

\section{Accelerated Acute Rejection}

Accelerated acute rejection occurs because of preformed cytotoxic anti-HLA antibodies. It was originally described before adequate crossmatch techniques and assays for antibodies were available. Humoral vascular rejection occurring immediately after reperfusion is called hyperacute rejection. Hyperacute rejection is an exceedingly rare event in the modern era but must still be considered when evaluating early graft dysfunction in patients who were highly sensitized before the transplant. Patients with hyperacute or delayed hyperacute rejection (occurring days after the transplant due to an an amnestic antibody response) may be febrile, and the kidney is usually swollen and tender upon palpation. Ultrasound may show impaired flow. Surgical exploration is indicated to exclude vascular compromise and obtain a biopsy to confirm the diagnosis.

\section{Acute T-Cell-Mediated Rejection}

Acute T-cell-mediated rejection before the end of the first week is uncommon, especially in the current era when most patients get antibody induction. It is usually asymptomatic and manifested by either a rise in serum creatinine or by a failure of renal function to improve in a patient with DGF. Ultrasound may show elevated resistive indices, but this finding is not specific. The diagnosis is made by a kidney biopsy showing interstitial infiltration with mononuclear cells and occasionally eosinophils (measured by the iscore), disruption of the tubular basement membranes by the infiltrating cells or tubulitis (t-score), and intimal arteritis (v-score) (Table 1).
Corticosteroids are the most common firstline treatment for episodes of acute cellular rejection. Intravenous methylprednisolone, 250$500 \mathrm{mg}$ daily for $3 \mathrm{~d}$ followed by tapering steroid doses is the usual practice. Most initial episodes of Banff type 1 acute T-cell-mediated rejection respond to corticosteroids and show improvement in graft function back to baseline levels. Treatment of acute cellular rejection with antithymocyte globulin is more effective in restoring kidney function and preventing graft loss than treatment with corticosteroids, but is also associated with more adverse effects. The efficacy of thymoglobulins, especially those of rabbit origin, has been clearly established for the treatment of steroid-resistant acute cell-mediated rejection (Webster et al. 2006). Banff grade IIIII patients show the greatest benefit with rejection reversal rates ranging from $88 \%$ to $97 \%$. The outcome after rejection depends on the severity of the rejection and the response to therapy. Return to within $15 \%$ of the baseline serum creatinine is associated with the best long-term prognosis. If kidney function does not return to baseline or if there is a new decline in function after successful treatment of an acute rejection, a repeat biopsy should be considered to rule out additional rejection or other causes of graft dysfunction.

\section{Acute Antibody-Mediated Rejection}

Acute antibody-mediated rejection (AMR) tends to occur early, particularly in patients who were presensitized, and often presents solely with elevated serum creatinine. Kidney biopsies showing histological features of endothelial damage including neutrophil infiltration, necrosis, apoptosis, and thrombosis in patients with detectable donor-specific antibody (DSA) in serum have traditionally been termed vascular rejection. The presence of circulating DSA has been shown to play a key role in these findings. The 2003 update of the 1997 Banff criteria defined AMR on the basis of three criteria: the presence of anti-HLA DSA in serum, allograft histology ( polymorphonuclear-rich glomerular and/or peritubular inflammation), and positive staining for C4d in peritubular capillaries. In 
S. Chandran and F. Vincenti

Table 1. Banff 2007 update of Banff 1997 diagnostic categories for renal allograft biopsies

\begin{tabular}{|c|c|c|}
\hline Diagnostic category & Description & Criteria \\
\hline \multicolumn{3}{|l|}{ 1. Normal } \\
\hline \multirow[t]{3}{*}{$\begin{array}{l}\text { 2. Antibody-mediated } \\
\text { changes }\end{array}$} & $\begin{array}{l}\text { C4d deposition without } \\
\text { morphologic evidence of } \\
\text { active rejection }\end{array}$ & $\begin{array}{l}\mathrm{C}^{+} \mathrm{d}^{+} \text {; circulating DSA present; no signs of acute or } \\
\text { chronic TCMR or ABMR }\end{array}$ \\
\hline & $\begin{array}{l}\text { Acute antibody-mediated } \\
\text { rejection }\end{array}$ & 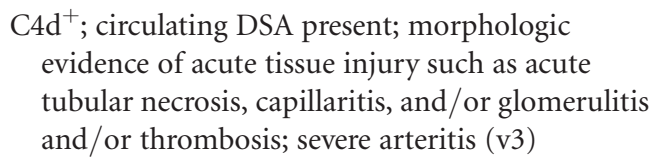 \\
\hline & $\begin{array}{l}\text { Chronic active antibody- } \\
\text { mediated rejection }\end{array}$ & $\begin{array}{l}{\mathrm{C} 4 \mathrm{~d}^{+} \text {; circulating DSA present; morphologic }}^{\text {evidence of chronic tissue injury such as }} \\
\text { glomerular double contours and/or peritubular } \\
\text { capillary basement membrane multilayering; IF/ } \\
\text { TA; fibrous intimal thickening in arteries }\end{array}$ \\
\hline 3. Borderline change & $\begin{array}{l}\text { "Suspicious" for acute T-cell- } \\
\text { mediated rejection }\end{array}$ & $\begin{array}{l}\text { No intimal arteritis }(\mathrm{v} 0) \text {, but there are interstitial } \\
\text { infiltrates and tubulitis insufficient to meet } \\
\text { criteria for T-cell-mediated rejection (i0-i1, t1-t3 } \\
\text { or i2-i3, } 1 \text { ) }\end{array}$ \\
\hline \multirow{4}{*}{$\begin{array}{l}\text { 4. T-cell-mediated } \\
\text { rejection (TCMR) }\end{array}$} & Acute T-cell-mediated rejection & Type I: i2-3, t2-3 \\
\hline & & Type II: v1-v2 \\
\hline & & Type III: v3 \\
\hline & $\begin{array}{l}\text { Chronic active T-cell-mediated } \\
\text { rejection }\end{array}$ & $\begin{array}{l}\text { Arterial intimal fibrosis with mononuclear cell } \\
\text { infiltration in fibrosis, formation of neo-intima }\end{array}$ \\
\hline $\begin{array}{l}\text { 5. Interstitial fibrosis } \\
\text { and tubular atrophy }\end{array}$ & $\begin{array}{l}\text { No evidence of any specific } \\
\text { etiology }\end{array}$ & $\begin{array}{l}\text { Severity graded I-III by the extent of } \\
\text { tubulointerstitial fibrosis and atrophy }\end{array}$ \\
\hline 6. Other & $\begin{array}{l}\text { Changes not considered to be } \\
\text { due to rejection }\end{array}$ & $\begin{array}{l}\text { For example, acute pyelonephritis, BK polyoma } \\
\text { virus interstitial nephritis }\end{array}$ \\
\hline
\end{tabular}

Data adapted from Solez et al. (2008).

2011, the Banff meeting identified significant limitations of $\mathrm{C} 4 \mathrm{~d}$ as a diagnostic marker for AMR and acknowledged the existence of C4dnegative AMR as a phenotype (Sis et al. 2010; Mengel et al. 2012). The diagnostic criteria continue to evolve.

The reported incidence of AMR varies worldwide from $3.1 \%$ to $40 \%$, depending on the diagnostic criteria, recipient sensitization, and the immunosuppressive regimen. Unlike cellular rejection, AMR responds poorly to corticosteroids and antithymocyte agents alone. The treatment of AMR often requires a combination of strategies that increase the clearance of circulating DSA and decrease antibody production (Vincenti et al. 2010). The most effective treatment so far appears to be a combination of plasmapheresis, IVIg, and rituximab, which is a monoclonal antibody to CD20 targeting B cells (Lefaucheur et al. 2009). Bortezomib, a pro- teasome inhibitor, directly targets plasma cells, although effects may be delayed because of the slow decay in levels of DSA. Eculizumab, a terminal complement inhibitor, has been used in refractory cases of AMR to arrest complementmediated injury while other therapies directed at DSA take effect.

Although knowledge of the diagnosis and pathophysiology of AMR is advancing, evidence supporting existing treatments is poor (Roberts et al. 2012). Treatment decisions regarding dosing and efficacy are often made on the basis of data from desensitizing protocols. However, the observed effects may differ when treating AMR in the posttransplantation setting owing to ongoing antigenic stimulation. Further studies are required to determine the optimal treatment of AMR and to gain a better understanding of the risk-benefit ratio because the adverse effects and cost of treatments are not insignificant. 


\section{Thrombotic Microangiopathy}

Posttransplant thrombotic microangiopathy (TMA) can present either de novo or as a recurrence in patients with atypical hemolytic uremic syndrome (HUS). Most patients with recurrent TMAwill eventually lose the graft. De novo posttransplant TMA is a rare but serious complication of transplantation, occurring in $0.8 \%-15 \%$ cases, and associated with graft loss in more than one-third of cases with systemic manifestations (Noris and Remuzzi 2010). Usually, de novo TMA occurs in the early posttransplant days, but it may also develop 2-6 yr after transplantation. The pathogenesis is not well understood, but it is speculated that the endothelial lesions caused by ischemia-reperfusion injury, viral infection, or rejection may be amplified by the endothelial injury caused by immunosuppressive agents such as calcineurin inhibitors (CNIs), which have direct prothrombotic and vasoconstrictive effects, or the TOR inhibitors, which are antiangiogenic. This form of TMA is commonly limited to the renal allograft and usually presents with renal dysfunction unaccompanied by thrombocytopenia or a hemolytic anemia. It is usually, but not always, reversible with discontinuation of the CNI or mTOR inhibitor. Other reported risk factors include transplantation from extended criteria donors, viral infections, antiphospholipid antibodies, complement regulation disorders, OKT3, and malignancy. TMA has also been associated with acute antibody-mediated rejection of the kidney transplant.

Treatment for de novo TMA has not been well defined, and even complete withdrawal of the $\mathrm{CNI}$ is associated with graft loss rates as high as $60 \%-100 \%$. Addition of plasma exchange has been reported to salvage the graft in $\sim 80 \%$ of cases. Switching the CNI to sirolimus may not lead to improvement because sirolimus itself has been associated with the development of posttransplant TMA. Cautious reintroduction of CNIs may be successful in those who recover graft good function. Replacement of CNIs and/ or mTOR inhibitors with belatacept, a secondgeneration CTLA4-Ig that blocks T-cell costimulation, is an alternative strategy for the treatment of posttransplant TMA that has been reported to be successful without precipitating acute rejection or recurrence (Ashman et al. 2009). Eculizumab, a high-affinity humanized anti-C5 monoclonal antibody that prevents deployment of the terminal complement system and generation of the cytolytic membrane attack complex, has been described as rescue therapy in severe or resistant cases of systemic posttransplant TMA associated with complement gene abnormalities or antibody-mediated rejection.

\section{THE FIRST YEAR}

\section{Recurrent Disease}

Recurrent primary kidney disease is the third most frequently reported cause for graft failure $10 \mathrm{yr}$ after kidney transplantation (Briganti et al. 2002; Canaud et al. 2012). The incidence of recurrent disease and resultant graft failure varies depending on the disease, duration of follow-up, whether or not the primary cause of ESRD was known, and the frequency of kidney biopsies. The most frequent recurrent diseases have been focal segmental glomerulosclerosis (FSGS), IgA nephropathy, diabetic nephropathy, membranoproliferative (MPGN) and membranous glomerulonephritis, hemolytic uremic syndrome/thrombotic thrombocytopenic purpura (HUS/TTP), and lupus nephritis. Recurrent disease may present as increased serum creatinine, new-onset or increased proteinuria, and/or hematuria. FSGS, HUS, and oxalosis may recur in the first few days to weeks posttransplantation, whereas the timing is variable but usually late in the others.

Idiopathic FSGS recurs in $20 \%-50 \%$ of cases, and in up to $80 \%$ if it has recurred in a prior kidney transplant. Risk factors for recurrence include age of onset of FSGS in the native kidneys between 6 and $15 \mathrm{yr}$, rapid course of the original disease, diffuse mesangial proliferation on histology, and non-African-American ethnicity. The strongest risk factor is recurrence in a previous transplant. Recurrent disease usually presents with nephrotic proteinuria. About $80 \%$ recur in the first $4 \mathrm{wk}$, and recurrence should be considered in all cases with delayed 
or slow graft function. The diagnosis is made by a kidney biopsy that shows foot process effacement on electron microscopy. Sclerosis may not be evident in early recurrence, and light microscopy may show normal glomerular architecture. Case reports and uncontrolled series have noted a substantial reduction in proteinuria after plasma exchange, possibly because of removal of circulating glomerular permeability factors. Predictors of response to plasma exchange include early recurrence and early initiation of treatment after recurrence. Unfortunately, proteinuria may recur after treatment and may require additional or even periodic, ongoing treatments. High-dose cyclosporine can induce remission of proteinuria and may be combined with plasmapheresis. A reduction in proteinuria with an ACE inhibitor and/or an ARB may also be attempted.

Recurrent IgA nephropathy is common after transplantation, with reported incidence varying from $13 \%$ to $53 \%$. Recurrence is more common among recipients of living related-donor transplants as compared with deceased donor transplants and may be associated with reduced graft survival. Maintenance steroid use has been associated with a reduced risk of recurrence. There is no effective therapy for preventing recurrent IgA nephropathy, although fish oil, ACE-Is, and ARBs frequently reduce proteinuria and possibly preserve kidney function.

Membranous nephropathy ( $\mathrm{MN}$ ) recurs in $\sim 40 \%$ of patients after kidney transplantation. Autoantibodies to the phospholipase A2 receptor (PLA2R) are found in $>70 \%$ of those with idiopathic $\mathrm{MN}$, and are associated with recurrence. Treatment with rituximab stabilized both the proteinuria and serum creatinine, and circulating anti-PLA2R became undetectable. The recurrence rate in idiopathic type I MPGN is $20 \%-30 \%$ and exceeds $80 \%$ in type 2 disease. The introduction of eculizumab, a terminal complement inhibitor, may provide the first effective therapy for this disease.

The risk of posttransplant recurrence of atypical HUS depends on the genetic abnormality involved and ranges from $15 \%$ to $20 \%$ in patients with gene mutations for membrane cofactor protein to $50 \%-100 \%$ in patients with gene mutations of circulating complement regulators such as factor $\mathrm{H}$. Recurrence usually develops within $4 \mathrm{wk}$. Most patients develop microangiopathic anemia, thrombocytopenia, and kidney dysfunction, whereas others present with rapidly progressive graft dysfunction without showing the classic hematologic manifestations. Long-term graft survival is $\sim 30 \%$ lower in those with recurrence. Treatment strategies have included plasmapheresis, IVIg, and rituximab. Eculizumab has recently emerged as an effective treatment for recurrent HUS.

Both ANCA-associated vasculitis and antiGBM disease have low rates of recurrence if the disease is quiescent at the time of transplant. Most cases occur beyond the first posttransplant year with no significant impact on allograft function. Relapses of ANCA-associated vasculitis usually manifest as pauci-immune necrotizing glomerulonephritis, but graft function can also be affected by acute arteritis, ureteral stenosis, and obstructive uropathy due to granulomatous vasculitis. Pretransplantation disease course, disease subtype, ANCA type or titer, time of transplantation, or donor type does not predict recurrence. Kidney ANCA-associated vasculitis generally responds well to high-dose prednisolone and cyclophosphamide. Other treatment modalities that have been tried include MMF, plasmapheresis, IVIg, and rituximab. Clinical recurrence of anti-GBM disease is rare and consists of isolated case reports only. The incidence may be higher in those with circulating anti-GBM antibody at the time of transplantation. Treatment of clinically active anti-GBM disease may include pulse steroids, cyclophosphamide, and plasma exchange, particularly if there is potentially lifethreatening pulmonary involvement.

Primary hyperoxaluria is caused by deficiency of hepatic peroxisomal alanine:glyoxylate aminotransferase, leading to increased synthesis and urinary excretion of oxalate, recurrent calcium oxalate urolithiasis, and nephrocalcinosis. In renal failure, insoluble oxalates accumulate throughout the body, especially in bone and arteries. Oxalate overproduction persists following isolated kidney transplantation, leading to recurrent calcium oxalate deposition in 
$>90 \%$ of transplanted kidneys and eventually graft loss. Total body oxalate burden is often very high in patients with advanced chronic kidney disease, and plasma and urine oxalate levels may remain high for months and years, respectively, even in patients undergoing simultaneous kidney and liver transplantation. Early posttransplant urinary dilution through aggressive fluid administration, and early and frequent dialysis in those with DGF, are essential to minimize the effects of hyperoxalemia and hyperoxaluria. In the long term, measures designed to increase oxalate excretion and reduce production include the maintenance of urine output $>3 \mathrm{~L} / \mathrm{d}$, and the use of alkaline citrate, neutral phosphate, and magnesium oxide. Pharmacological doses of pyridoxine may also be helpful in reducing hyperoxaluria in some patients, especially those with a Gly170Arg mutation.

\section{BK Virus}

Polyomavirus BK (BKV) is ubiquitous among the general population, establishing a latent, asymptomatic infection in immunocompetent individuals. The cellular reservoir of latent BKV infection comprises numerous cell types, including urothelial cells. The adaptive immune response and, in particular, $\mathrm{T}$ cells play a crucial role in the clearance of most viral infections, and the development of both cellular and humoral immunological memory is important in the immune response against BKV.

In kidney transplant recipients, BKV reactivation is common and leads to nephropathy (BKVN) in $\sim 5 \%$ of recipients within a year after transplantation. Rarely, infection has been noted to cause ureteral stenosis. Patients with BKVN generally present with an asymptomatic decrease in renal function. Although the development of BKVN has been linked in some studies to specific immunosuppressive agents such as tacrolimus, mycophenolate mofetil, polyclonal anti-T-cell antibodies, and corticosteroid pulses, it is likely that the cumulative intensity of the immunosuppressive regimen rather than one specific agent increases the risk for BKVN. Other factors such as cold ischemia time, cadaveric donor, and the number of HLA mis- matches and rejection episodes have been inconsistently associated with BKVN (Vasudev et al. 2005).

The renal disease spectrum begins with viruria and sequentially proceeds through viremia and tubulointerstitial nephritis before ending in extensive irreversible kidney damage. Intervention at an early stage is therefore of paramount importance to prevent graft loss. Screening for active BKV replication involves the detection of viral DNA by quantitative PCR in urine and in blood and, in some cases, the detection of BKVinfected "decoy cells" or aggregates of BKV virions, the so-called haufen in urine. The diagnosis of BKVN can only be made by a kidney biopsy. Histopathological grades of severity have been defined as ranging from viral cytopathic changes of near-normal renal parenchyma and no or minimal tubular atrophy, interstitial fibrosis, or inflammation, to diffusely scarred renal tissue with extensive tubular atrophy, interstitial fibrosis, and inflammation. Because BKV affects the kidney in a random, multifocal manner, falsenegative biopsy results may occur, especially in early stages of the disease.

Currently, reducing immunosuppression is the only established mode of therapy and aims to restore the antiviral immune response. There is no standard strategy, although reducing or eliminating MMF is often the first step (Hirsch et al. 2005). Upon in vitro infection, BKV activates the intracellular PI3K/Akt/mTOR pathway, and sirolimus reduced LTAg expression in a dose-dependent manner. Clinical efficacy of mTOR inhibitors in treating BKVN has not been convincingly shown.

Beyond tapering and/or altering immunosuppression, other antiviral agents have been proposed. Although polyomaviruses do not express the known target of cidofovir, viral DNA polymerase, they showed in vitro inhibition of BKV. Unfortunately, randomized controlled trials are lacking, and several confounders including the concomitant tapering of immunosuppression complicate the interpretation of the clinical effectiveness of cidofovir. Known side effects include nephrotoxicity and, rarely, anterior uveitis. Treatment of BKV infection with IVIg, the pyrimidine synthesis inhibitor 
leflunomide, and fluoroquinolones have been reported to be successful in small case series.

\section{AFTER THE FIRST YEAR}

\section{Late Allograft Failure}

Chronic allograft failure is second only to death with a functioning graft as a cause of late renal graft loss after transplantation. Despite advances in transplantation reducing early acute rejection rates to $<15 \%$ and lifting $1-y r$ graft survival $>90 \%$, long-term graft attrition rates have remained unchanged at $4 \%$ loss per year (MeierKriesche et al. 2004). The most commonly reported pathological change is chronic interstitial fibrosis and tubular atrophy (IF/TA) accompanied by vascular changes and glomerulosclerosis. The term "chronic allograft nephropathy" or "CAN," initially coined as a general descriptor for fibrosis in the allograft representing the final pathway of nephron injury, was replaced in 2005 as per recommendations of the Banff Consensus Conference by more specific etiologically relevant diagnoses and the designation of nonspecific fibrosis as interstitial fibrosis and tubular atrophy (IF/TA), no cause identified (Solez et al. 2007). Moderate to severe $\mathrm{IF} / \mathrm{TA}$ is present in a quarter of recipients at $1 \mathrm{yr}$ after transplant, and in $\sim 90 \%$ by $10 \mathrm{yr}$ (Nankivell and Chapman 2006). It is believed to repre- sent the cumulative effect of chronic immuneand nonimmune-mediated injury to the graft.

Chronic rejection due to alloimmunity in the allograft may be cell or antibody mediated and is now recognized in the majority of patients with late graft loss (El-Zoghby et al. 2009). Although early data indicated a strong association between acute and chronic rejection, recent studies using classic histological and gene expression analysis show that early T-cell-mediated rejection has no impact on term outcome (Reeve et al. 2013). The degree of HLA mismatches, the presence of preformed or de novo DSA, and nonadherence to immunosuppressive medications have been shown to increase the risk of late graft loss. Chronic transplant glomerulopathy and arterial changes of accelerated graft arteriosclerosis, especially with lymphocytes in the fibrosing intima, splintering of the internal elastic lamina, and formation of neomedia and neo-intima inside the vessel, are reliable features of chronic rejection (Fig. 3). The presence of markers of ongoing injury in allografts in the form of active lymphocytic tubulitis, arteritis, or positive peritubular capillary C4d staining, even in the context of significant fibrosis appears to be more deleterious to the graft than bland fibrosis alone (Fig. 4) (Mannon et al. 2010).

Nonimmunological factors associated with late allograft failure include increased donor
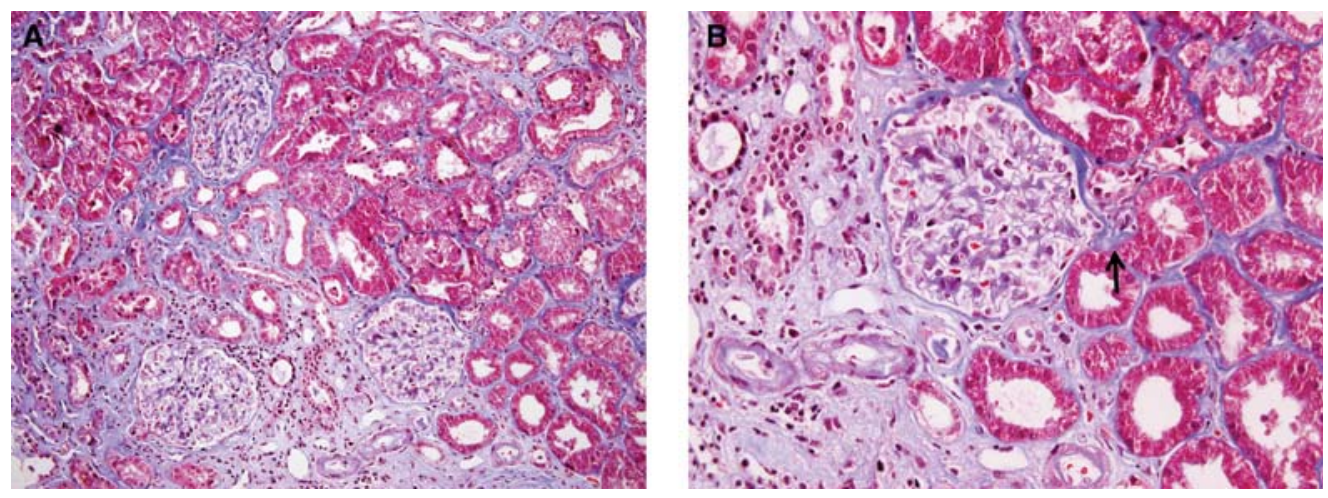

Figure 3. Pathological lesions on kidney allograft biopsies associated with chronic calcineurin inhibitor nephrotoxicity. (A) Areas of interstitial fibrosis and tubular atrophy alternating with preserved tubulointerstitium produce a picture of "striped fibrosis." (B) Peripheral arteriolar hyalinosis (arrow). Masson's trichrome stain. (Images courtesy of Dr. Zoltan Laszik.) 
A

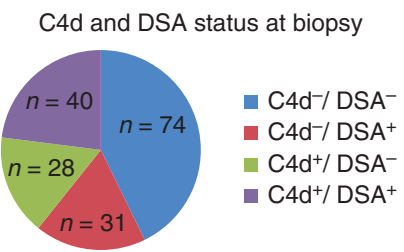

B Renal allograft survival at 12 months postbiopsy

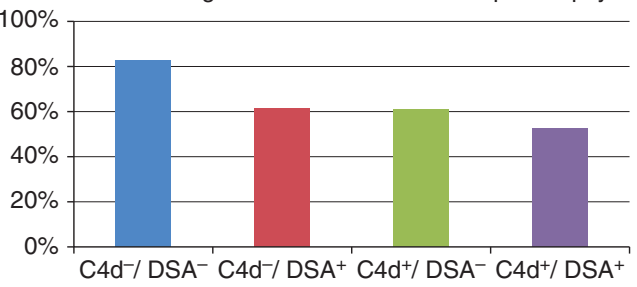

Figure 4. Impact of presence or absence of C4d and donor-specific antibody (DSA) on kidney allograft survival after for-cause biopsy. Both C4d positivity and the presence of detectable DSA increased the relative risk of graft failure with the best prognosis in the $\mathrm{C}_{4} \mathrm{~d}^{-} / \mathrm{DSA}^{-}$group and the worst graft survival in the $\mathrm{C} 4 \mathrm{~d}^{+} / \mathrm{DSA}^{+}$group. (Figures created from data in Gourishankar et al. 2010.)

age, receipt of an ECD kidney, nephrotoxicity from CNIs, and systemic disorders such as diabetes, hypertension, and dyslipidemia. Histological features suggestive of CNI toxicity include obliterative arteriolopathy, ischemic collapse or scarring of the glomeruli, vacuolization of the tubules, and focal areas of tubular atrophy and interstitial fibrosis producing a picture of "striped" fibrosis (Fig. 5). The extent of IF has been correlated to the expression of transforming growth factor- $\beta$, which is up-regulated by CNIs. However, morphological lesions attributed to chronic CNI toxicity are nearly universal in long-functioning grafts, and it has been difficult to establish a definitive causal link, particularly in the modern era with the use of lower doses of CNIs as compared with when they were first introduced (Stegall et al. 2011). Therefore, the diagnosis of probable CNI toxicity is usually one of exclusion. Interestingly, the Deterioration of Kidney Allograft Function (DeKAF) study suggested that immune-mediated injury rather than CNI toxicity may be a more significant contributor to graft loss than previously thought; and in this study, patients with a histological diagnosis of CNI nephrotoxicity were found to be less likely to lose their allografts than those without such a diagnosis (Gourishankar et al. 2010).

Clinically, there is declining graft function, often accompanied by proteinuria and hypertension. A kidney biopsy is useful in determining whether specific features of immune-mediated injury are present and to rule out reversible causes of graft dysfunction such as BKVN, acute rejection, or recurrent kidney disease. When de-
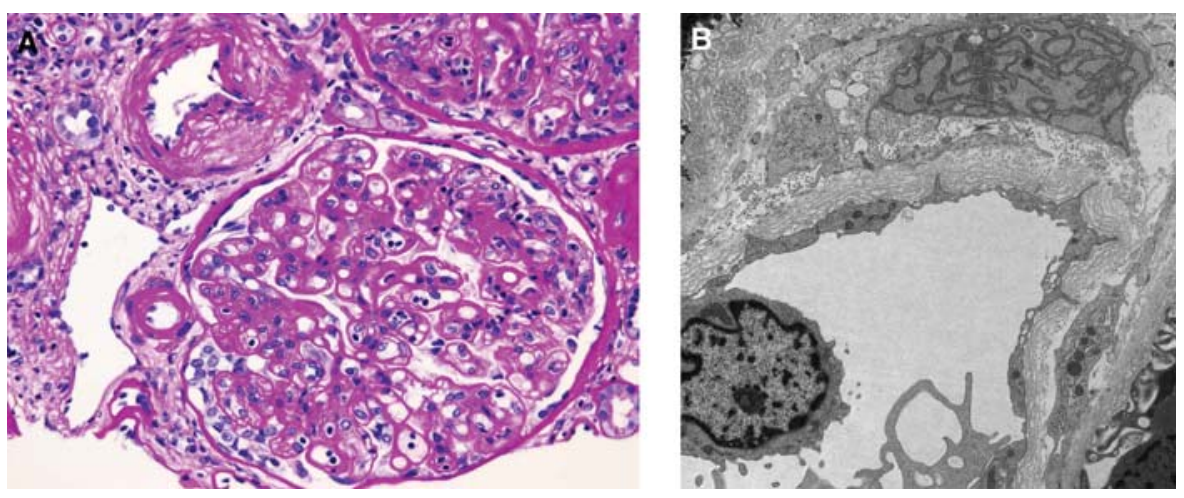

Figure 5. Pathologic lesions on kidney allograft biopsies characteristic of chronic antibody-mediated rejection. (A) Double contoured glomerular basement membranes accompanied by increased mesangial matrix and prominent glomerulitis. Periodic acid Schiff stain. (B) Peritubular capillary basement membrane multilayering on electron microscopy. (Images courtesy of Dr. Zoltan Laszik.) 
teriorating function from CNI nephrotoxicity arises in low-immune-risk patients and ongoing immune injury is excluded, CNI withdrawal and maintenance with concentration-controlled mycophenolate mofetil and corticosteroids have been shown to improve GFR with a small risk of rejection and graft loss. In those with evidence of CNI toxicity and high immune risk, either CNI minimization or substitution with mTOR inhibitors can be considered, accompanied by appropriate monitoring. Augmentation of immunosuppression may be considered in patients whose biopsy shows chronic cell- or antibody-mediated rejection with the addition of ACE inhibitors or angiotensin-receptor blockers to reduce proteinuria. Plasmapheresis, IVIg, and rituximab have shown variable results, and benefits from bortezomib and eculizumab are unproven in this population.

\section{Pregnancy after Kidney Transplantation}

Kidney transplantation improves reproductive function in women with ESRD, often restoring fertility within a few months. Data from large voluntary registries and a recent meta-analysis (Deshpande et al. 2011) indicate overall live birth rates of $71 \%-79 \%$ among pregnant kidney transplant recipients. Despite this success, pregnancy poses risks to both fetus and graft, and thus, preconception counseling, family planning, and contraception are critical aspects of posttransplant care. A higher rate of obstetrical complications including miscarriage, gestational diabetes, preeclampsia, Cesarean section delivery, preterm labor, and low birth weight is seen in kidney transplant recipients. The transplant kidney is neither affected by, nor does it affect, a vaginal delivery. Younger maternal age is associated with favorable outcomes. Pregnant kidney transplant recipients should be preferably referred to an obstetrician with expertise in managing high-risk pregnancies.

Acute rejection rates of $3 \%-10 \%$ have been reported during pregnancy, although the impact of pregnancy on graft survival remains controversial, with several studies showing no statistically significant difference when compared with matched nonpregnant controls. Prepregnancy hypertension, elevated serum creatinine, and proteinuria have been associated with adverse graft and fetal outcomes. Extrapolating from studies of pregnant women with chronic kidney disease, an estimated GFR $<40 \mathrm{~mL} / \mathrm{min}$ per $1.73 \mathrm{~m}^{2}$ and proteinuria $>1 \mathrm{~g} / \mathrm{d}$ are considered to be risk factors for accelerated decrease in GFR and potentially graft loss. KDIGO guidelines suggest waiting for at least $1 \mathrm{yr}$ after transplantation before becoming pregnant and only attempting pregnancy when kidney function is stable with $<1 \mathrm{~g} / \mathrm{d}$ of proteinuria.

There are no prospective data on the safety of immunosuppressive agents during pregnancy. Mycophenolate is reported to cause severe structural malformations, and a characteristic phenotype includes cleft lip and palate, microtia, and absent external auditory canals. In September 2012, the FDA approved a single shared Risk Evaluation and Mitigation Strategy (REMS) for all mycophenolate-containing prescription drugs. The goals of the mycophenolate REMS are to prevent an unplanned pregnancy in patients using the drug, to minimize fetal exposure, and to inform patients about the serious risks associated with this class of medications. The REMS includes a medication guide, training for healthcare professionals, and the establishment of a pregnancy registry for women who become pregnant and agree to participate in the registry.

Despite the FDA category D, azathioprine has been used safely over the years in pregnant transplant recipients. Thus, mycophenolate is generally changed to azathioprine, and a 6-wk window is recommended between discontinuing mycophenolate and attempting conception. Because of evidence of embryotoxicity in animal studies of sirolimus, it is recommended that mTOR inhibitors be discontinued or replaced before pregnancy is attempted. The incidence of fetal malformations has not been found to be higher in women taking cyclosporine. CNIs are usually continued during pregnancy with careful monitoring and dose adjustment as needed because drug levels can fall with the increase in plasma volume. Prednisone at doses low enough to prevent thymic aplasia (usually $<15 \mathrm{mg} / \mathrm{d}$ ) 
is considered safe in pregnant kidney transplant recipients.

\section{REFERENCES}

Annual Data Report of the Scientific Registry of Transplant Recipients. 2011. http://srtr.transplant.hrsa.gov/annual _reports/2011 (accessed May 1, 2013).

Ashman N, Chapagain A, Dobbie H, Raftery MJ, Sheaff MT, Yaqoob MM. 2009. Belatacept as maintenance immunosuppression for postrenal transplant de novo drug-induced thrombotic microangiopathy. Am J Transplant 9: 424-427.

Briganti EM, Russ GR, McNeil JJ, Atkins RC, Chadban SJ. 2002. Risk of renal allograft loss from recurrent glomerulonephritis. N Engl J Med 347: 103-109.

Canaud G, Audard V, Kofman T, Lang P, Legendre C, Grimbert P. 2012. Recurrence from primary and secondary glomerulopathy after renal transplant. Transpl Int 25: 812-824.

Deshpande NA, James NT, Kucirka LM, Boyarsky BJ, Garonzik-Wang JM, Montgomery RA, Segev DL. 2011. Pregnancy outcomes in kidney transplant recipients: A systematic review and meta-analysis. Am J Transplant 11: 2388-2404.

El-Zoghby ZM, Stegall MD, Lager DJ, Kremers WK, Amer H, Gloor JM, Cosio FG. 2009. Identifying specific causes of kidney allograft loss. Am J Transplant 9: 527-535.

Gourishankar S, Leduc R, Connett J, Cecka JM, Cosio F, Fieberg A, Gaston R, Halloran P, Hunsicker L, Kasiske B, et al. 2010. Pathological and clinical characterization of the "troubled transplant": Data from the DeKAF study. Am J Transplant 10: 324-330.

Hirsch HH, Brennan DC, Drachenberg CB, Ginevri F, Gordon J, Limaye AP, Mihatsch MJ, Nickeleit V, Ramos E, Randhawa P, et al. 2005. Polyomavirus-associated nephropathy in renal transplantation: Interdisciplinary analyses and recommendations. Transplantation 79: $1277-$ 1286.

Irish WD, Ilsley JN, Schnitzler MA, Feng S, Brennan DC. 2010. A risk prediction model for delayed graft function in the current era of deceased donor renal transplantation. Am J Transplant 10: 2279-2286.

Kayler LK, Srinivas TR, Schold JD. 2011. Influence of CITinduced DGF on kidney transplant outcomes. Am J Transplant 11: 2657-2664.

Lefaucheur C, Nochy D, Andrade J, Verine J, Gautreau C, Charron D, Hill GS, Glotz D, Suberbielle-Boissel C. 2009. Comparison of combination plasmapheresis/IVIg/antiCD20 versus high-dose IVIg in the treatment of antibody-mediated rejection. Am J Transplant 9: 1099-1107.

Li L, Khatri P, Sigdel TK, Tran T, Ying L, Vitalone MJ, Chen A, Hsieh S, Dai H, Zhang M, et al. 2012. A peripheral blood diagnostic test for acute rejection in renal transplantation. Am J Transplant 12: 2710-2718.

Mannon RB, Matas AJ, Grande J, Leduc R, Connett J, Kasiske B, Cecka JM, Gaston RS, Cosio F, Gourishankar S, et al. 2010. Inflammation in areas of tubular atrophy in kidney allograft biopsies: A potent predictor of allograft failure. Am J Transplant 10: 2066-2073.
Meier-Kriesche HU, Schold JD, Srinivas TR, Kaplan B. 2004. Lack of improvement in renal allograft survival despite a marked decrease in acute rejection rates over the most recent era. Am J Transplant 4: 378-383.

Mengel M, Sis B, Haas M, Colvin RB, Halloran PF, Racusen LC, Solez K, Cendales L, Demetris AJ, Drachenberg CB, et al. 2012. Banff 2011 Meeting Report: New concepts in antibody-mediated rejection. Am J Transplant 12: $563-$ 570.

Muthukumar T, Dadhania D, Ding R, Snopkowski C, Naqvi R, Lee JB, Hartono C, Li B, Sharma VK, Seshan SV, et al. 2005. Messenger RNA for FOXP3 in the urine of renalallograft recipients. N Engl J Med 353: 2342-2351.

Nankivell BJ, Chapman JR. 2006. Chronic allograft nephropathy: Current concepts and future directions. Transplantation 81: 643-654.

Noris M, Remuzzi G. 2010. Thrombotic microangiopathy after kidney transplantation. Am J Transplant 10: 15171523.

Pleass HC, Clark KR, Rigg KM, Reddy KS, Forsythe JL, Proud G, Taylor RM. 1995. Urologic complications after renal transplantation: A prospective randomized trial comparing different techniques of ureteric anastomosis and the use of prophylactic ureteric stents. Transplant Proc 27: 1091-1092.

Reeve J, Sellarés J, Mengel M, Sis B, Skene A, Hidalgo L, de Freitas DG, Famulski KS, Halloran PF. 2013. Molecular diagnosis of T cell-mediated rejection in human kidney transplant biopsies. Am J Transplant 13: 645-655.

Roberts DM, Jiang SH, Chadban SJ. 2012. The treatment of acute antibody-mediated rejection in kidney transplant recipients-A systematic review. Transplantation 94: 775-783.

Siedlecki A, Irish W, Brennan DC. 2011. Delayed graft function in the kidney transplant. Am J Transplant 11: 22792296.

Sis B, Mengel M, Haas M, Colvin RB, Halloran PF, Racusen LC, Solez K, Baldwin WM 3rd, Bracamonte ER, Broecker V, et al. 2010. Banff '09 Meeting Report: Antibody mediated graft deterioration and implementation of Banff working groups. Am J Transplant 10: 464-471.

Solez K, Colvin RB, Racusen LC, Sis B, Halloran PF, Birk PE, Campbell PM, Cascalho M, Collins AB, Demetris AJ, et al. 2007. Banff '05 Meeting Report: Differential diagnosis of chronic allograft injury and elimination of chronic allograft nephropathy ("CAN"). Am J Transplant 7: $518-526$

Solez K, Colvin RB, Racusen LC, Haas M, Sis B, Mengel M, Halloran PF, Baldwin W, Banfi G, Collins AB, et al. et al. 2008. Banff 07 classification of renal allograft pathology: Updates and future directions. Am J Transplant 8: 753760.

Stegall MD, Park WD, Larson TS, Gloor JM, Cornell LD, Sethi S, Dean PG, Prieto M, Amer H, Textor S, et al. 2011. The histology of solitary renal allografts at 1 and 5 years after transplantation. Am J Transplant 11: 698-707.

Vasudev B, Hariharan S, Hussain SA, Zhu YR, Bresnahan BA, Cohen EP. 2005. BK virus nephritis: Risk factors, timing, and outcome in renal transplant recipients. Kidney Int 68: 1834-1839.

Veale JL, Yew J, Gjertson DW, Smith CV, Singer JS, Rosenthal JT, Gritsch HA. 2007. Long-term comparative outcomes 
S. Chandran and F. Vincenti

between two common ureteroneocystostomy techniques for renal transplantation. J Urol 177: 632-636.

Vincenti F, Cohen SD, Appel G. 2010. Novel B cell therapeutic targets in transplantation and immune-mediated glomerular diseases. Clin J Am Soc Nephrol 5: 142-151.
Webster AC, Pankhurst T, Rinaldi F, Chapman JR, Craig JC. 2006. Monoclonal and polyclonal antibody therapy for treating acute rejection in kidney transplant recipients: A systematic review of randomized trial data. Transplantation 81: 953-965. 


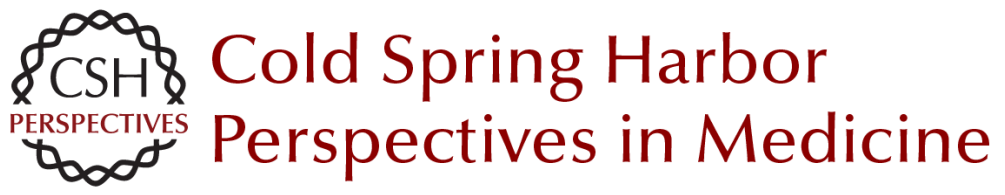

\title{
Clinical Aspects: Focusing on Key Unique Organ-Specific Issues of Renal Transplantation
}

\author{
Sindhu Chandran and Flavio Vincenti
}

Cold Spring Harb Perspect Med 2014; doi: 10.1101/cshperspect.a015644 originally published online December 26, 2013

\section{Subject Collection Transplantation}

Heart Transplantation: Challenges Facing the

Field

Makoto Tonsho, Sebastian Michel, Zain Ahmed, et al.

Bioethics of Organ Transplantation Arthur Caplan

Overview of Clinical Lung Transplantation Jonathan C. Yeung and Shaf Keshavjee

Immunological Challenges and Therapies in Xenotransplantation Marta Vadori and Emanuele Cozzi

Clinical Aspects: Focusing on Key Unique Organ-Specific Issues of Renal Transplantation Sindhu Chandran and Flavio Vincenti

T-Cell Costimulatory Blockade in Organ

Transplantation Jonathan S. Maltzman and Laurence A. Turka

Regulatory T-Cell Therapy in Transplantation: Moving to the Clinic Qizhi Tang and Jeffrey A. Bluestone

Opportunistic Infections--Coming to the Limits of Immunosuppression?

Jay A. Fishman
Overview of the Indications and Contraindications

for Liver Transplantation

Stefan Farkas, Christina Hackl and Hans Jürgen Schlitt

Facial and Hand Allotransplantation Bohdan Pomahac, Ryan M. Gobble and Stefan Schneeberger

Induction of Tolerance through Mixed Chimerism David H. Sachs, Tatsuo Kawai and Megan Sykes

Pancreas Transplantation: Solid Organ and Islet Shruti Mittal, Paul Johnson and Peter Friend

Tolerance--Is It Worth It? Erik B. Finger, Terry B. Strom and Arthur J. Matas

Lessons and Limits of Mouse Models Anita S. Chong, Maria-Luisa Alegre, Michelle L. Miller, et al.

Effector Mechanisms of Rejection Aurélie Moreau, Emilie Varey, Ignacio Anegon, et al.

The Innate Immune System and Transplantation Conrad A. Farrar, Jerzy W. Kupiec-Weglinski and Steven H. Sacks

For additional articles in this collection, see http://perspectivesinmedicine.cshlp.org/cgi/collection/ 